\title{
TTR
}

Traduction, terminologie, re?daction

\section{Traduire les Antilles en anglais : trahir ou fêter la polyphonie?}

\section{Valérie Budig-Markin et Martha Manier}

Volume 13, numéro 2, 2e semestre 2000

Les Antilles en traduction

The Caribbean in Translation

URI : https://id.erudit.org/iderudit/037413ar

DOI : https://doi.org/10.7202/037413ar

Aller au sommaire du numéro

Éditeur(s)

Association canadienne de traductologie

ISSN

0835-8443 (imprimé)

1708-2188 (numérique)

Découvrir la revue

Citer cet article

Budig-Markin, V. \& Manier, M. (2000). Traduire les Antilles en anglais : trahir ou fêter la polyphonie? TTR, 13(2), 107-125. https://doi.org/10.7202/037413ar

\section{Résumé de l'article}

Traduire les Antilles en anglais : Trahir ou fêter la polyphonie? — Les rapports complexes de langue-culture aux Antilles, lorsqu'on les traduit du français vers l'anglais, se situent sur les plans culturel, naturel et créole-francais. Quand on traduit l'espagnol de Porto Rico en anglais, il s'agit surtout de problèmes d'emprunts, de transfert et de code switching anglais-espagnol dans l'original. Dans les deux cas, par contre, la même question se pose pour le traducteur: comment fêter la polyphonie sans la trahir? Nous examinons quatre nouvelles martiniquaises et portoricaines et leur unicité de contexte pour offrir des solutions spécifiques, des expressions exemplaires, des textes et contextes problématiques, et aussi pour signaler certains problèmes difficiles que pose la traduction des Antilles.
Tous droits réservés (C) TTR: traduction, terminologie, rédaction — Les auteurs, 2000
Cedocument est protégé par la loi sur le droit d'auteur. L'utilisation des services d'Érudit (y compris la reproduction) est assujettie à sa politique d'utilisation que vous pouvez consulter en ligne.

https://apropos.erudit.org/fr/usagers/politique-dutilisation/ 


\section{Traduire les Antilles en anglais : trahir ou fêter la polyphonie?}

\section{Valérie Budig-Markin et Martha Manier}

Dans La littérature franco-antillaise: Haiti, Guadeloupe et Martinique, Régis Antoine décrit la littérature francophone 1980-1994 spécifique à la Martinique et à la Guadeloupe comme "ensemble problématique, qui se développe parmi les bouleversements économiques et écologiques contemporains, les migrations transatlantiques, la conquête de lectorats nouveaux en France métropolitaine comme dans l'ensemble du monde francophone" (Antoine, 1992, p. 351). Les anglophones aux États-Unis ne profitent pourtant pas de traductions de ces ouvres si elles ne figurent pas parmi les plus rentables pour les maisons d'édition américaines. Le devoir échoit donc aux traducteurs indépendants de traduire les cuvres les plus récentes et moins consacrées, surtout la nouvelle, genre moins apprécié que la poésie, le roman, et même le conte dans le discours critique, surtout avant les années 1980 (Fonkoua, 1992, pp. 68, 69). Dans son aperçu de la nouvelle antillaise pour Notre Librairie, Rumuald Fonkoua classe plusieurs nouvelles selon les catégories d'une juxtaposition multiple comprenant réalisme/merveilleux, "nouvelleœuvre/nouvelle-texte » (p.73), passé/contemporanéité, et Afrique/ Antilles. À sa liste de nouvellistes, nous ajoutons une romancière et nouvelliste des années 1990, Suzanne Dracius, qui prendrait sa place parmi les Martiniquais Joseph Zobel, Raphaël Confiant, Claude Relouzat, Richard Labetian, Yva Léro, Xavier Orville, et ceux qui ont publié leurs nouvelles dans la revue martiniquaise Carbet et dans la Revue Présence Africaine, entre autres.

En traduisant en anglais les nouvelles de Dracius, des textes où s'entremêlent divers styles, cultures, et époques qui renvoient toujours à la créolité, plusieurs concepts de l'Éloge de la créolité, en particulier ceux de "l'interlangue " et "l'interlecte ${ }^{1}$ " exprimés par Bernabé, Chamoiseau, et Confiant, chevauchent ceux du traducteur.

${ }^{1}$ L'interlecte est, selon l'Éloge de la créolité, la rencontre de langues dans le même espace. 
Quoique son travail soit de dehors plutôt qu'à l'intérieur de la créolité, il fait face comme les Antillais à la transmutation, la transgression, le pouvoir des illusions, l'acte d'échange, et « la gestion [(ir)responsable] de l'espace linguistique » (Bernabé et al., 1995, p. 49). Selon les auteurs de l'Éloge, l'emploi exclusif du français de France aux Antilles pose le danger d'une langue idolâtrée qui interdirait l'acte littéraire et mènerait à la mort esthétique (pp. 47-48), tandis que le créole, disent-ils, exprime un trésor de particularités culturelles et linguistiques, tout en "frémissant de la vie du Tout-monde " (p. 51), expression de Glissant. Or, le français antillais, " interlangue " entre le français de France et le créole, propose une poétique multi-langue qui rompt l'ordre linguistique et contredit parfois des significations établies, sans avoir encore subi la traduction dans aucune autre langue, notamment l'anglais. Si la communicabilité de l'interlangue est difficile dans sa forme originelle, comment l'effectuer en traduisant vers l'anglais? Bernabé, Chamoiseau et Confiant présentent le modèle idéal pour toute traduction en décrivant l'usage " fécond " de l'interlecte, c'est-à-dire, "le maximum de communicabilité compatible avec l'expression extrême d'une particularité » (p. 52). Dans les deux cas, il faudrait harmoniser et préserver les diversités d'un monde "diffracté mais recomposé », ce que les auteurs de l'Éloge ont nommé « la diversalité » (p. 54). L'idée de la traduction en tant que diversalité rappelle l'exemple proposé par la protagoniste écrivaine Marie-Sophie dans le roman de Chamoiseau, Texaco.

Chamoiseau exprime la problématique d'écrire (et nous dirions aussi de traduire) l'interlecte martiniquais dans Texaco quand MarieSophie essaie de capturer par écrit la vie de son père Esternome, celle du quartier de Texaco, celle de sa propre vie à elle, en écrivant comme débutante en français. "Comment y ramener mon Esternome tellement créole? ... Je mesurais le gouffre " dit-elle (Chamoiseau, 1992, p. 412). Elle continue : «J'étais raccrochée à cette cathédrale (c'est-à-dire, ses souvenirs les plus sublimes) que je sauvais en moi et perdais du même coup » (p. 412). Son lecteur au français « impeccable », Ti-Cirique, rejette les tournures créoles dans l'écriture de Marie-Sophie pour un élitisme métropolitain, parlant de la capacité du français de capturer l'universel. Mais, le rappelle Marie-Sophie, il admirait aussi la façon dont « le frottement du merveilleux et du réel (comme pratiqué en Haïti depuis nanni-nannan) ajoutait aux approches des vérités humaines " (pp. 415-416). Ce que cherche Marie-Sophie, sans avoir beaucoup lu elle-même, est une écriture qui raconte la vraie expérience de la vie dans le temps, incorporée à la concrétisation de l'imagination artistique :

[...] une écriture informée de la parole, et des silences, et qui reste vivante, qui bouge en cercle et circule tout le temps, irriguant sans cesse de vie ce qui a été écrit avant, et qui réinvente le cercle à chaque fois comme le font les spirales qui sont à tout moment dans le 
futur et dans l'avant, l'une modifiant l'autre, sans cesse, sans perdre une unité difficile à nommer $[\ldots]$ (p. 413)

Marie-Sophie, tout comme le traducteur des Antilles, est traductrice de cultures, mettant en juxtaposition la culture créole et la française, le passé, le présent et l'avenir. Dans son écriture, dans celle de Chamoiseau et dans celle de Susanne Dracius, la frontière de l'interlecte entre le créole et le français de Martinique rend déjà difficile la traduction de cultures dans la norme de l'identité de la majorité qui rencontre la « contra-identité » de résistance minoritaire (Budick, 1996, pp. 11, 15). Pour le traducteur en anglais, l'altérité se dédouble.

Quelques problèmes assez élémentaires de la traduction du français de Martinique vers l'anglais sont la terminologie de la nature, celle de la nourriture, l'expression linguistique des figures culturelles, le créole cité dans le texte français, et les couches de langue de différentes classes socio-économiques de chaque micro-culture.

En Martinique la montagne Pelée, géant aux flancs verts parsemés d'orchidées sauvages, surveille l'île qu'elle domine : les villages à l'intérieur de l'île, les côtes et leurs villes aux deux extrêmes de cette partie du nord de lî́le, les champs de l'ancienne habitation de canne à sucre tout près de la falaise où le dernier Caraïbe s'est jeté dans le vide plutôt que de vivre sans liberté. La réalité naturelle, l'histoire et le mythe colorent la littérature martiniquaise, dont la nouvelle toute récente de Suzanne Dracius, "La Montagne de Feu», et ses deux nouvelles traduites en anglais, " De sueur, de sucre et de sang " et « La Virago ». S'il s'agit dans la première de la vie autour d'une distillerie de rhum il y a un siècle, dans la deuxième c'est plutôt la jeunesse et la vitesse modernes de Fort-de-France. Mais le poids de l'histoire et de la tradition y pèse presque autant, et le discours maintient une complexité qui est aussi difficile à traduire.

Dans " De sueur, de sucre et de sang" les mots tels que "pied-bois" et " poux-bois », et "lambi », aussi communs qu'ils soient dans la description du paysage antillais, sont absents du Petit Robert. La traduction mot à mot du francais martiniquais en « françaisde-France » selon le modèle " scientifique ${ }^{2}$ » (Robinson 1991, pp. 6667) est possible, donnant à lire : " arbre ", " termites », " coquille", mots qui se traduisent de nouveau en anglais comme "tree", " termites", et "conch ", le rythme se perdant dans la double traduction.

${ }^{2}$ En nous servant du concept de la traduction " scientifique », nous voulons dire le modele comprenant les perspectives de Thomas d'Aquin et d'Augustin, entre autres, modèle qui cherche à comprendre les choses objectivement, dans leur totalité. 
L'onomatopée se traduit de deux façons différentes. Si le lecteur anglophone peut reconnaître le sens dans le son du mot original, il ne faut pas le traduire, puisque l'original est préféré : le pipiri (oiseau matinal qui chante au lever du jour : " little bird that sings at dawn ») et le yen-yen (moustique : " mosquito ") sont peut-être compréhensibles pour l'anglophone, surtout s'ils sont assez bien contextualisés et devraient rester en français martiniquais en italique.

Dans son Subversive Scribe: Translating Latin American Fiction, Suzanne Jill Levine propose une autre traduction de l'onomatopée dans un contexte cubain, là où un mot son-sens dans le texte original peut être trahi et fêté en même temps avec un nouveau son-sens dans la traduction: l'allitération «se abalanza balanceándose " en espagnol se traduit, par exemple, en un mot créé qui porte la même sorte de son-sens en anglais, "balanswaying "; et un mot créé "peligrotescamente " en espagnol cubain devient encore une fois un nouveau son-sens "strangerously" inventé par Levine (Levine, 1991, p. 17). Dracius se sert souvent de l'exclamation, "Tchip! ", mot créé dont le son-sens est méprisant. Dans " La Virago », la narratrice (à la voiture grise) réfléchit à la scène dont elle est spectatrice :

- Mais ne serait-ce pas " ma " Virago Yamaha ? A cette distance, comment savoir? Je n'y connais rien en motos... Tchip ! Qu'est-ce que j'en ai à faire, après tout, de cette moto rouge ? (Dracius, 1995, p. 77 , nos caractères gras)

Le traducteur peut garder le mot pour son parfum culturel ou choisir un autre mot son-sens, comme "Hmph!», expression de mépris en anglais. Le son du "tchip " est différent et fait penser au chant d'un oiseau, plus léger et plein de verve, comme les protagonistes de l'auteur, mais le son-sens en anglais porte le sens d'une personne qui, comme la narratrice, se classifie comme "assise " ou plutôt passive, s'opposant à l'explosion de vie et de bruit de la personne qui fait corps avec sa Virago.

Un mot mettant en relief l'interlecte créole-français tel que «zwezo » est avant tout un son de la nature figuré par un mot dont l'orthographe est plutôt créole, quoiqu'il imite aussi le son du mot français et signifie comme lui " oiseau». La double traduction en français-de-France et puis en anglais risque de perdre la richesse multilangue, multi-classe et multi-culture du mot, et l'élégance et la sensualité de l'original seraient au mieux maintenues en gardant le mot original ou en choisissant une espèce d'oiseau local de cette région dont le son du nom est rythmique et l'orthographe rappelle le créole.

D'autres termes de la flore régionale invitent à une approche différente : tel est le " fleurit-six-mois », par exemple, un buisson dont le nom latin utilisé en anglais est « photinia ». Or, l'importance de cette 
plante dans la nouvelle est surtout son rôle de symbole de la vie de la protagoniste, rouge de passion et de vie pendant une première époque de floraison, et puis verte, calme, trop calme, le rouge étant fané. Le nom en français portant le sens de cette période fleurie, ainsi que la beauté de la langue française, peuvent bien être transmis en le laissant en français en italique, si le traducteur croit que ses lecteurs comprendront les mots apparentés de "fleur ", "six", et " mois ». On a aussi recours à la traduction mot-mot qui a l'air objective-scientifique à l'Augustin mais qui répond aussi aux exigences de Goethe, une traduction dialectique, vivante (Robinson, 1991, pp. 78-81) ${ }^{3}$, car " flowers-six-months » garde toutes les possibilités de l'imagination du lecteur, une caractéristique idéale de la traduction selon Levine.

En ce qui a trait à la nourriture et à la cuisine, chaque microculture est assez unique quand elle est décrite en français (ou en espagnol), ainsi qu'en créole. La pistache en Martinique est la cacahuète à laquelle on a donné le nom de pistache, un aliment que la plupart des Martiniquais n'ont guère l'occasion de grignoter, selon Dracius, soit parce que c'est un produit plus cher, soit parce qu'elle est importée d'ailleurs. Si « pistache » est traduit par " peanut », le mot n'a plus le sens satirique et solidaire de l'original sans une note d'explication culturelle.

Le concept se complique encore dans le juron martiniquais, "Sacrée pistache !" qui se traduit selon le modèle mot-mot par " Blasted peanut !", ou plutôt par " Damn it! " selon le modèle senssens $^{4}$. La traduction sens-sens perd tout élément culturel et toute connotation régionale; il faut donc garder le "Blasted peanut! », choisissant le compromis afin de maintenir le sens dialogique du goût du produit (plus ou moins) local en sacrifiant l'ironie dans le rapport cacahuète/pistache, pour ne pas trop céder au choix facile qui risque de diluer le parfum de la « diversalité ».

C'est le cas en général avec la nourriture : il faut laisser un peu à l'imagination et garder en même temps l'association de sens physiques, rapprochant les plats, les goûts, les ingrédients ou les éléments culturels de Martinique et de la vie du lecteur, pour inviter

\footnotetext{
${ }^{3}$ Robinson résume le troisième niveau de traduction selon Goethe : « It is not enough to oppose the system; you have to contaminate system with life. You have to inject frozen dualism with time, delta t, change over time, progress. If truth is found dynamically emerging from an ongoing dialectical process, Goethe says, then it is alive, then it is a living organism that enlivens everyone who dedicates him — or herself to its emergence » (p. 81).

${ }^{4} \mathrm{Ce}$ que nous appelons le modèle sens-sens ( juxtapose au modèle mot-mot scientifique, est le modèle proposé par Martin Luther dans la traduction de la Bible. C'était un modèle dont le but était la conversion du lecteur, qui ne s'intéressait pas autant aux mots mais plutôt au message des textes.
} 
une réaction souvent physique chez lui. Les " accras de morue ", par exemple, vont plus flatter le palais d'un public spécifique de lecteurs en anglais si on les traduit par "fish balls ", quelque chose dont ils peuvent imaginer le goût. Encore pour les tenter par des goûts et parfums qu'ils reconnaissent, le "blaff " se traduit comme "spicy citron fish bouillon ". Le traducteur prête attention aux mots tels que " kassav ", qui n'est pas un melon comme en anglais mais plutôt une galette de manioc - et actuellement aussi un groupe de musiciens de zouk, d'ailleurs. Le mot " shrubb " se traduit de façon sens-sens pour donner une idée du goût d'écorces de mandarines ou d'oranges macérées dans du rhum. Le ti-punch porte le goût, la tradition, et l'élément de quelque chose de petit et pourrait se traduire comme " shot of rum and lime ", avec une note sur la structure linguistique et d'autres exemples communs : le ti-nain (petite banane à cuire) et le ti-bois (celui qui accompagne le joueur de tambour en tapant avec des baguettes).

Dracius se sert d'autres mots rares ou nouveaux en français dans ses nouvelles : " brillantiné », " vaseliné ", " encravaté » et " tafiateuse ", par exemple, ce dernier qui dénomme celle qui boit du rhum ou du " tafia » en français créolisé, tandis que les premiers sont forgés à partir des noms propres bien connus des anglophones et révèlent, ensemble avec " encravaté » un effort d'imiter et plaire à leurs maîtres, mais " encravaté " n'est pas "cravaté » tel qu'il s'écrit dans $L e$ Petit Robert, et les laboureurs, comme l'interlangue les décrivant, sont différents, gauches, pauvres, et de trop. Si les premiers adjectifs se traduisent par "Brillantined » et " Vaselined », des participes passés imitant les noms propres à leur origine, la traduction d'« encravaté(s) " devrait être aussi gauche que ces personnages timides, "shirted and tied »ce qui ajoute une idée de leur désir d'échapper à cette rencontre étouffante aussitôt que possible.

Il faut laisser les expressions aux connotations historiques multiples et importantes en créole dans le nouveau texte, avec leur traduction en anglais. "Béké ", " marron ", " Ni krik, ni krak », et "Kimafoutiésa!" transmettent un marronnage moderne, une résistance linguistique et culturelle en Europe et aux États-Unis caractérisée par la diversité, la relation et l'opacité, plutôt que par la pensée linéaire, la filiation et la transparence (Glissant, 1990, ch. 5). Ce qui est derrière les traductions de " white plantation owners (and their descendants) 》 et " maroons, or escaped slaves 》 est aussi important qu'il est complexe; l'origine de « marron », par exemple, est l'espagnol (" cimarrón ") devenu un créole espagnol-français antillais et réunit hispanophones, Créoles et francophones en solidarité et dans une histoire pareillement triste. L'expression « Ni krik ni krak » réfère à la stichomythie « Krik ! - Krak ! » qui termine un conte oral, un passage ou un épisode de celui-là et demande une réponse des auditeurs, et transporte toute une histoire d'oralité et de griots d'Afrique aux Antilles. Toute l'expression créole, "Kimafoutiésa!», est incorporée dans la traduction de « La Virago », suivie par la traduction en français, 
"Qui m'a foutu ça !" pour montrer le rapport linguistique, le lien argotique et la résistance créole-française, puis s'ajoute en anglais, "Who fed me that crap! " pour en partager le dialogue entre les liens linguistiques et culturels. Il faudrait transmettre le sens de l'argot qui exprime le dégoût de ce vieux diable qui hante les mornes « depuis la mort du dernier Caraibe et la descente en ville du dernier Nègre Marron ", un esprit réveillé de son sommeil séculaire par le bruit d'une motocyclette.

Le personnage du vieux diable fait partie de la nouvelle « La Virago 5 " et c'est lui qui donne un coup de pied à la narratrice à la fin de l'histoire :

Réveillé de son sommeil séculaire par le vacarme de la moto, le vieux diable qui hante le Gros-Morne depuis la mort du dernier Caraïbe et la descente en ville du dernier Nègre Marron a trouvé tellement scandaleuse toute cette histoire de mâles-femmes motorisées, de bougres salaces, d'amitiés féminines naissantes, qu'il m'a donné un grand coup de pied au derrière pour avoir osé la conter.

- Sacrée pistache! Kimafoutiésa ! Qui m'a foutu à présent une telle qualité d'espèces de petites femelles wayayay dévergondées tout bonnement ? grogna-t-il en créole-dragon avant de se rendormir, éccuré par les temps nouveaux et le côté déluré des Martiniquaises d'aujourd'hui, - non sans avoir bu un petit feu de rhum agricole, pour oublier :

- Ki zafè champagne ésa ? Qu'est-ce qu'elles ont avec leur champagne? Le rhum, c'est ça qui est bon même, a-t-il décrété en bâillant... (p. 78)

Si dans la nouvelle le coup de pied exprime un rejet de l'histoire de la conteuse narratrice, selon Dracius, le vieux diable, tel qu'elle l'avait imaginé, l'a en fait poussée à écrire encore et plus sérieusement ${ }^{6}$. Il parle un créole-dragon plutôt rude juxtaposé à un français assez fin, rappelant à la narratrice et l'auteur la couche de tradition et de valeurs culturelles que sous-entend la littérature martiniquaise. Il est difficile de la traduire en français de France, puis de la traduire encore une fois en anglais. Et telle est la raison pour laquelle, en rédigeant cette nouvelle pour une publication éventuelle dans une anthologie en français pour étudiants universitaires aux États-Unis, la maison d'édition Houghton Mifflin, de Boston, était prête à accepter de la publier à condition qu'on enlève de la nouvelle tout le passage ci-

${ }^{5}$ « La Virago » existe en deux versions. La version entière, que l'auteur date du 14 juillet 1993 de Casablanca, nous est parvenue de Dracius non publiée. La version raccourcie se trouve dans notre DIVERSITE: La nouvelle francophone à travers le monde (Boston, Houghton Mifflin, 1995), pp. 74-78.

${ }^{6}$ Conférence de Dracius à Humboldt State University, Arcata, en Californie, avril 1998. 
dessus comprenant le personnage du vieux diable, parce que ni son apparition, ni son commentaire ni son langage ne correspondaient aux attentes linguistiques, littéraires et culturelles des lecteurs (marchands) anglophones. "L'autre ", donc, n'est pas aussi désiré qu'on pourrait croire : pas trop " autre », en effet, pour ne pas risquer de perdre quelques ventes éventuelles. Houghton Mifflin a publié la nouvelle, nue, coquine, simple et linéaire comme il faut, mais sans sa raisond'être culturelle et historique. La limite de la traduction est la censure dans l'omission de passages entiers, là où une autre culture est rejetée du fait d'être différente. La conclusion originale de "La Virago », ce palimpseste du passé, du créole, du Nègre Marron, a pris forme visible encore une fois dans la traduction anglaise de la nouvelle lue à l'université de Humboldt State au moment de la visite de l'auteur? Traduite de sa forme originale, elle est plus compréhensible comme nouvelle d'origine caraïbe, de loin plus appréciée pour sa structure complexe de conte rappelant l'oralité et le conte d'Afrique qu'un anglophone peut reconnaître et comparer au conte et aux « leprechauns " d'Irlande, par ailleurs. En fait, de tout le texte de la nouvelle "La Virago ", le public a aimé discuter surtout de cette conclusion surprenante qui introduit un nouveau personnage et tout un nouveau cadre structurel à la fin de l'histoire, la rendant opaque et complexe, plutôt qu'un système binaire - femme/homme, jeune/vieux, moderne/traditionnel, urbain/rural, étranger/indigène, indépendant/ respectueux des aïeux et individuel/collectif - qui se résoud en linéarité, comme une comédie européenne. Tout est là où les éditeurs américains voudraient éliminer en français-créole ce qui est trop original, qui exige trop d'explications, trop de mots, trop de risque de gêner un public qui préfère lire ou vendre le banal dit " accessible » et " bien écrit ".

Dans sa Poétique de la relation, Glissant caractérise la culture antillaise et ses rapports de "relation " plutôt que de linéarité comme dans "l'Ouest ", qui trouve son origine dans le principe de la création unique du monde à la base du judéo-christianisme, par exemple, et du darwinisme, quoique différemment. La complexité du concept de relation en Martinique s'exprime de façon symbolique dans « De sueur, de sucre et de sang " à l'égard des concepts de famille, de race, et de classe sociale. Tout un paragraphe explique le rapport entre les "B » de Saint-Pierre, la lignée de la famille mulâtre en l'honneur de qui une statue a été érigée en son honneur sur la place centrale, la lignée familiale qui se comporte d'une façon assez paternaliste et condescendante envers l'autre, celle de Fort-de-France, qui a pourtant plus d'argent. Emma, qui ne s'y intéresse pas beaucoup, est, paraît-il, d'une classe sociale assez proche pour faire une épouse convenable à

7 Traduction non publiée de Valérie Budig-Markin, «The Virago ", lue en lecture simultanée avec l'original lu par l'auteur, Suzanne Dracius, Humboldt State University, Arcata, Californie, avril, 1998. Voir la bibliographie pour une autre traduction, celle de Doris Y. Kadish et Jean-Pierre Piriou. 
Émile B. La classe sociale dépend aussi du sexe et de l'âge, puisqu'un parrain éventuel de la famille ne va pas accepter son rôle si l'enfant est une fille. La traduction de la parole d'Émile va aussi exiger un ton condescendant et paternaliste qui manque à celle de sa femme Emma. Le monologue intérieur se traduit d'ailleurs selon la classe sociale, changeant par exemple quand la cuisinière Man Sonson donne son avis. Mais quand elle le donne indirectement à travers des commentaires de la narratrice, il faut choisir judicieusement entre les niveaux linguistiques des différentes couches sociales mélangées et proférées indirectement. Comprenant le grec et connaissant de près les pensées d'Emma, la narratrice est d'une classe sociale peut-être assez près de celle de sa protagoniste, même peut-être un membre de sa famille. Les travailleurs de la Distillerie qu'Emma écoute attentivement tous les jours font évidemment partie de la classe la plus basse représentée dans la nouvelle. Ils ne montent à la maison du Maître $\mathrm{B}$ que cette seule fois où, tout "brillantinés, vaselinés, encravatés " (Dracius, 1992, p. 35), ils viennent offrir leurs félicitations aux nouveaux mariés, s'éclipsant aussitôt qu'ils sont venus. Membres d'une classe maintenue à une certaine distance d'Emma, ils sont plutôt des corps irisés de sueur mais qu'elle écoute assez parler pour pouvoir reconnaître leurs voix individuelles; leurs paroles différentes, néanmoins, proposent tout un autre système de différences riches en nuances et en variété. Toutes ces voix, directes et indirectes, exigent leur propre registre linguistique, dont le créole et le français qui y trouvent leurs places différentes.

La question de classe sociale fait partie de toute traduction, mais aux Antilles et dans une nouvelle de complexité de voix comme "De sueur, de sucre et de sang", les détails de cette différence multiple sont importants. Traduit-on l'eau de cologne "Étoile ", par exemple? C'est une marque très bon marché en Martinique qui établit la couche sociale, mais les anglophones ne la reconnaîtront évidemment pas, et pour eux le français donne un air d'élégance contraire à l'impression souhaitée; "Star of Martinique " clarifie et établit le compromis de classe, d'artefact culturel et de rythme.

Toutes les phrases en créole se maintiendront dans la nouvelle interlangue, mais il faudrait pouvoir reconnaître la différence entre le créole du vieux qui connaît bien les cyclones, celui du rêveur, et celui du maillot mouillé, peut-être en ajoutant une phrase ou deux pour indiquer celui qui parle à un moment donné. Le créole bégayé se traduit en imitant le changement de structure du mot créole dans le mot en anglais; "ka-kabèche " devient "hea-head": "Sa ou ni an kakabèche ou, nèg? ${ }^{8}$ serait "But what's in that hea-head of yours, man? ». La traduction en anglais ne se bégaie pas facilement sans "stop " phonétique, mais normalement on ne lit pas la traduction à

\footnotetext{
${ }^{8}$ Dracius le traduit en français : « Mais qu'est-ce que t'as dans le cra-crâne, mon vieux? » (Dracius, 1992, p. 36).
} 
voix haute. Le mot « kabèche » se reconnaît, d'ailleurs, comme un mot d'origine romane - «caput » en latin, "cabeza » en espagnol, et "cabeça " en portugais; l'origine espagnole ou portugaise de "kabèche" accentue le fait que le créole martiniquais n'est pas forcément ou toujours une interlangue avec le français, qui est ici un chaînon manquant. En considérant la question de niveau de langue et son rapport à une classe sociale en anglais, finalement, nous envisageons la possibilité, sans vouloir considérer le phénomène de façon trop réductrice, d'avoir recours à la syntaxe d'une langue largement répandue de la diaspora noire, comme celle des Noirs aux États-Unis. Il reste à déterminer l'efficacité d'un tel projet. La multiple hiérarchisation linguistique et sociale dont les règles sont strictes et bien comprises par les divers participants rappelle le code switching, l'une des clés de la traduction aux Antilles de l'espagnol.

Le traducteur de l'espagnol fait souvent face à des textes bilingues dans les Antilles, surtout à Porto Rico, où l'interlecte entre l'espagnol et l'anglais exprime une réalité où sont intimement liés classe, culture et lexique à la fois caraïbes et (nord-)américains. Les nouvelles d'Ana Lydia Vega éclatent de complexités et d'obstacles pour le traducteur. Ses nouvelles "Pollito/Chicken" du recueil de 1983 Vírgenes $y$ mártires et " Historia de arroz con habichuelas » sont pour leur part des traductions de la société de son Porto Rico natal. Dans ces deux œuvres, ainsi que dans plusieurs autres de ses fictions, Vega préconise la libération féminine, l'indépendance du territoire portoricain du gouvernement des États-Unis, et la fin éventuelle des préjugés de race des peuples de l'île. Elle déplore la déchéance des traditions et pratiques locales, la société de consommation qui y prend racine, et le dédoublement de la personnalité des peuples de l'île, exprimés dans l'épigraphe en français qui ouvre la nouvelle « Pollito/Chicken » : «Un homme à cheval sur deux cultures est rarement bien assis " (Albert Memmi). L'épigraphe d'« Arroz " encourage les Portoricains à travailler ensemble sans permettre que l'orgueil personnel brise les efforts de l'ensemble : « Oh, Familia Singular,/ en dación entregada/ sin el orgullo/ que rompe/ los nobles esfuerzos... " (Lolita Lebrón) [Oh, Famille Sacrée,/ livrée à l'acte de donner/sans l'orgueil/ qui rompt/ les nobles efforts...] Le ton élevé s'accorde avec l'emploi de l'allégorie comme histoire mais contraste avec les concurrents allégoriques choisis : le riz et les haricots, d'un côté (dans une assiette), et les hotdogs de l'autre (dans une autre assiette), tous les trois des plats nationaux des basses classes servis de façon simple. Les premiers sont des éléments culturels de base à Porto Rico, qui se trouvent en opposition aux hot-dogs, un produit non-définissable du fast food des États-Unis. Leur lutte représente celle des Portoricains qui vise le renversement de l'impérialisme yankee. 
Or, dans les deux nouvelles, le nom de l'auteur de l'épigraphe rappelle leur action politique et leur philosophie libératrice : Albert Memmi, philosophe, sociologue et humaniste tunisien, qui s'oppose à l'oppression des Arabes, des Juifs, et des femmes, et Lolita Lebrón (dont l'allitération du nom n'est pas une coïncidence) qui a confronté le Congrès des Etats-Unis, une bombe à la main, en 1954, demandant l'indépendance de son île natale. Le sous-texte ou plutôt le "surtexte" imposé de façon subliminale à la nouvelle, accentue sa subversion linguistique et culturelle.

Porto Rico, qui s'appelait Boricua avant l'arrivée des Européens de différentes nations (Colomb en 1493, Ponce de León en 1508 , et Drake en 1595), était habité par les peuples indigènes arawak et caraïbe. Ces peuples exploités furent décimés, et les Européens instituèrent l'esclavage pour se constituer une main-d'œuvre. Après une série de mouvements révolutionnaires, les Portoricains acquirent leur indépendance de l'Espagne en 1897, un an seulement avant la guerre hispano-américaine de 1898, à la suite de laquelle le Traité de Paris légitima l'occupation de Porto Rico, de Cuba et des Philippines par les États-Unis. En 1917, les Portoricains sont devenus citoyens des ÉtatsUnis, mais ce n'est qu'en 1952 que l'île a eu son propre gouverneur et une Constitution en tant que "état libre associé ». Un mouvement indépendantiste, dont Vega est ,partisane, continue à réclamer la séparation de Porto Rico des États-Unis, mais la majorité de la population n'a pas voté en faveur de cette séparation. Le lien culturel le plus important entre les États-Unis et Porto Rico est la population portoricaine de New York.

Dans «Pollito/Chicken », Vega met l'emphase sur le " nuyorkino ", le Portoricain qui habite à New York. Son personnage Suzie Bermiudez épelle son prénom et son nom de famille déjà à l'américaine : " $z$ » dans "Suzie », comme Suzie Parker, une Américaine bien connue à l'époque, et "iu 》 dans «Bermiudez » imitant la phonétique américaine plutôt que l'espagnole. Une habitante portoricaine de New York depuis une dizaine d'années, selon Vega, Suzie appartient à une classe sociale connue sous le nom de " pitiyanqui ». Traduit du " petit Yankee » en français, le terme porte une connotation malheureuse dans l'œuvre de Vega. Cette étiquette de la basse classe provient d'une élite socialement et intellectuellement cultivée, ayant étudié le français dont elle se sert comme langue de culture. Ce groupe des élites qui préfère l'indépendance de Porto Rico en surnomme un autre qui aime mieux rejoindre le colonisateur, une classe sociale qui a du mal à joindre les deux bouts. Selon Gerald Guiness, le pitiyanqui dans la nouvelle est un Portoricain né dans l'île qui « imitates, admires, flatters and sometimes fawns over this North American neighbor » (Guiness, 1984, p. 36) [(qui) imite, admire, flatte et se met parfois à plat ventre devant ce voisin d'Amérique du nord], peu importe où habite le pitiyanqui, que ce soit à Porto Rico ou à New York. Le pitiyanqui est une classe sociale et la langue parlée par cette 
classe est un patois où l'anglais et l'espagnol cohabitent, se juxtaposent et s'entremêlent dans des combinaisons différentes. Quelle est la structure de cet interlecte " pitiyanqui » et quels problèmes pose-t-elle pour la traduction? Comment les résoudre? Les linguistes diraient que le pitiyanqui réunit différents modèles linguistiques, y compris le "code switching" anglais-espagnol, l'emprunt de l'anglais, et le transfert des mots et de la syntaxe étrangers de la langue dominante (anglais), ce qui donne à l'espagnol portoricain un « accent étranger».

Les critiques ont souvent raté leur cible en caractérisant le discours du pitiyanqui, d'abord en disant que cette langue, peut-être en sa qualité de colonisée, est une langue " impure " (Vélez, 1986, p. 70). L'argument opposé de Shana Poplack est beaucoup plus convaincant (et moins ethnocentrique). Suzie (et alors la narratrice aussi) se sert du code switching, et cette alternance entre l'anglais et l'espagnol suggère, selon Poplack, que ce personnage a appris les deux langues couramment, au plus tard dans son adolescence. Elle s'exprime avec l'expertise linguistique d'une jeune femme qui a fait ses études secondaires. Son emploi du langage montre un transfert rapide et correct entre grammaires linguistiques et culturelles exprimées de façon parfaitement bilingue (Poplack, 1982, p. 237). D'autres critiques disent que Suzie ne se comprend pas et ne sait pas non plus où aller ou qui elle est. Mais elle agit en effet en comprenant sa situation assez bien et désire simplement une meilleure vie que celle qu'elle pourrait avoir en habitant à Porto Rico, retournant toutefois visiter un Porto Rico plus moderne, plus colonisé, plus assimilé - comme elle, d'ailleurs - à la société de consommation des États-Unis. Suzie est aussi une femme qui se sent encore des liens avec Porto Rico et essaie de maintenir des contacts avec l'íle. Mais sa réaction vient de la publicité touristique plutôt que de ses sentiments authentiques : "Lo que la decidió fue el breathtaking poster de Fomento que vio en la travel agency del lobby de su building. El breathtaking poster mentado representaba una pareja de beautiful people holding hands en el funicular del Hotel Conquistador» (Vega, 1981, p. 75). La phrase est traduite par, "Prompting her decison was the breathtaking poster of Fomento which she saw in the travel agency in the lobby of her building. The aforementioned breathtaking poster showed a couple of beautiful people holding hands in the Hotel Conquistador's cable car "). Finalement, on a mal compris la conclusion de la nouvelle. Quelques critiques croient voir dans son exclamation orgasmique provoquée par l'alcool, la fantaisie, et dans les attentions du barman noir une déclaration politique en faveur de l'indépendance portoricaine. En fait, en retombant dans le système discursif de la basse classe, où on substitue le $\mathrm{L}$ liquide pour le $\mathrm{R}$, en disant, "Viva Puelto Rico LIBREEEEEEEEEEE! », Suzie se mettrait plutôt du côté d'une classe sociale qui préfère que Porto Rico regagne la liberté et l'égalité d'un cinquante et unième état. Mais de toute façon, ce n'est pas un moment pour de telles réflexions! Tout en rencontrant dans son hôtel de luxe à Porto Rico le passé qu'elle avait tant essayé de refouler - la basse 
classe, son origine métisse, une grand-mère infirme et raciste, des boissons au maîs sucrées et bon marché - la liberté est quand même la sienne : elle a réussi à naviguer sur cette mer des Caraibes, ces eaux difficiles de la tension entre deux sociétés pleines d'attentes aux ÉtatsUnis et à Porto Rico, mais pour un moment, comme une Portoricaine de la basse classe, elle peut tout oublier, se détendre et s'amuser pleinement, ou un peu, au moins, chez elle. Le traducteur devrait-il pouvoir communiquer ces nuances en anglais, ou est-ce que tout dépend de la perspicacité du lecteur? Levine proposerait de laisser ouverte la fenêtre de l'imagination chez le lecteur de la traduction, pour garder cette qualité du texte original. (Voir ci-dessous la discussion du tagging.)

L'un des problèmes qu'on affronte comme traducteur est la traduction de ces multiples idées et idéaux pas même bien compris par les " experts", issus d'une culture essentiellement monolingue et monoculturelle aux États-Unis. Dans sa Poétique de la relation, Glissant considère justement une telle culture, une communauté ethnique majoritaire à la perspective individualisée et linéaire. Il en résulte un point de vue réducteur où les membres de la culture s'attendent à une transparence qui bloque en effet une compréhension de l'opacité d'une multi-culture (Glissant, 1990, ch. 5) comme celle des Antilles.

Au niveau du choix de langues qu'il faut traduire, le traducteur peut tout traduire dans une seule langue et signaler l'aspect bilingue du texte avec des expressions en italique - «Prompting her decison was the breathtaking poster of Fomento which she saw in the travel agency in the lobby of her building " - mais cette traduction perd le sens de transfert rapide et correct que Suzie et sa narratrice ont formulé, la perfection de la double grammaire qui devrait être maintenue, et le rythme saccadé des langues entretissées. Une autre approche est celle d'expliquer dans une note au lecteur que le texte original est produit dans deux langues exprimées en alternance, presque simultanément, mais ce choix ne peut jamais rendre claires ni la vraie nature ni les complexités de la langue, ni la personnalité, la vie, le point de vue culturel ou les motifs des personnages. Le traducteur pourrait, comme compromis, finalement, laisser un des mots en espagnol que ses lecteurs reconnaîtraient assez facilement: " Prompting her decisión was the breathtaking poster of Fomento which she saw in the travel agency in the lobby of her building $"$. Ce dernier choix donne le parfum de l'emploi simultané des deux langues, mais passe à côté de la dépendance de Suzie envers les deux langues pour sa communication, là où une langue est aussi importante que l'autre, les deux non différenciées dans son discours.

Dans la nouvelle « Historia de arroz con habichuelas », «A Story of Rice and Beans ", se déroule une lutte de pouvoir entre des aliments culturels dans la cuisine d'un restaurant. Sur le plan 
linguistique l'auteur a recours au transfert, traduisant un mot ou une structure de locution anglaise en une expression plutôt espagnole selon des modèles phonologiques de la langue espagnole :

$$
\begin{aligned}
& \text { "fildei "- " feel day 》(field day) } \\
& \text { " Japi Jordó "- "'Appy'otdog " (Happy Hotdog) } \\
& \text { " mistel »- - intruder " (mister) }
\end{aligned}
$$

Dans la traduction en anglais de "fildei » ou de "Japi Jordo ", il y a deux approches phonétiques : ou 1) essayer de produire une mauvaise prononciation similaire à l'anglais, comme " feel day » et " "Appy 'otdog " ou 2) maintenir l'original pour son son, puisque ces termes-ci ont d'autres connotations (" feel day») et indiquent un anglais d'Angleterre plutôt que des États-Unis ("'appy 'otdog»). Les mauvaises prononciations dans le contexte de la nouvelle même sont d'une importance minimale étant donné leur nombre (2); pourtant, si le narrateur ou le personnage principal prononçait des mots pour créer un effet comique ou pour caractériser celui qui parle, plus de soin serait nécessaire dans la traduction des sons, dans le choix de mots de la version anglaise. Il est amusant de retraduire de l'espagnol ce qui est venu de l'anglais, a pris un nouveau sens en espagnol, et doit se traduire différemment maintenant pour "fermer le cercle" du retour en un anglais qui porte le nouveau poids culturel de son hôte intérimaire.

Dans la cuisine, le riz appuie les haricots pour se défendre du saucisson hot-dog afin de ne pas être oubliés devant le nouveau résident populaire. Le sieur " místel », de « mister », désigne de façon méprisante le saucisson envahisseur. Le mot " místel " montre l'accent en anglais et encore une fois le remplacement de $R$ par $L$ chez les locuteurs du prolétariat. Quoique le sens du mot « místel » soit clair, il ne se lie pas logiquement aux saucissons dans une traduction en anglais sans rajouter d'autres mots pour réaliser le lien, ce qui casse malheureusement le rythme de la prose. Comme compromis, nous avons choisi le mot " intruder ", sa singularité maintenant le rythme et mettant l'accent sur les attributs socio-culturels du hot-dog comme signe linguistique des États-Unis.

L'emprunt est permis quand il n'y a pas de mot dans la langue cible qui exprime le sens communiqué, quand le concept ou l'objet n'existe pas. Par exemple, dans « Pollito/Chicken" Suzie Bermiudez prend un taxi de l'aéroport à son hôtel; pendant le trajet elle voit des " condominios» et des "shopping centers", emprunts de l'anglais. Pendant les années 60 et 70 l'essor du centre commercial venait de commencer aux États-Unis et les immeubles en copropriété se construisaient dans des quartiers touristiques pour les visiteurs et les locataires, contrairement aux "pisos" qui représentaient une plus grande stabilité grâce à leur achat par des familles qui y demeuraient. Un autre exemple d'emprunt se trouve dans la description peu flatteuse des Portoricains que fait Suzie : 
Se hubiera casado con algún drunken bastard de billar, de esos que nacen con la caneca incrustada en la mano y encierran a la fat ugly housewife en la casa con diez screaming kids entre los cellulitic muslos mientras ellos hacen pretty-body y le aplana la calle a cualquier shameless bitch. (Vega, 1981, p. 76)

"Ellos hacen pretty body" (Ils font joli corps) fait penser soit aux hommes musclés posant sur scène soit encore habillés d'un pantalon étroit et d'une chemise ouverte. Comme il s'agit dans la phrase d'une femme retenue chez elle avec dix enfants pendant les sorties du père, nous avons choisi de garder la deuxième image : convertir les hommes en un seul représentant " who shows off his pretty body and roams the streets 》 (qui aime bien exhiber son joli corps et erre dans les rues). Dans ce cas-ci l'emprunt continue, parce que le mot " pretty » ajoute une autre dimension à la description et fait contrepoids à l'image de « fat ugly housewife » en anglais dans l'original.

On emprunte aussi dans le cas du barman et ses copains, qui traînent dans le vestibule de l'hôtel tous les jours à reluquer les clientes. Vega invente le mot « hangueadores », provenant de l'anglais «hangers-on». Bien qu'il ait le suffixe -dor pour indiquer une personne, il ne se conforme pas aux règles de prononciation espagnole, selon lesquelles le $h$ muet en espagnol serait aspiré comme en anglais. "Tarzánico », un adjectif dont Vega se sert pour décrire la poitrine du barman, est un emprunt à la fois culturel et linguistique, important parce que Suzie met beaucoup de foi dans la culture populaire, soit le cinéma, soit la littérature, soit la mode américaine.

Ceux qui ont subi l'influence du colonialisme empruntent concepts et mots parce qu'ils n'ont pas d'équivalent dans la culture colonisée. Un transfert comprend un emprunt conceptuel renchéri d'une mise au point phonétique appropriée; il a lieu entre mots ou expressions, ici de l'anglais en espagnol portoricain. Dans une forme linguistique encore plus complexe, le code switching, une substitution spécifique et linguistiquement correcte $s$ 'insère entre des propositions entières et des phrases fragmentaires. Ce phénomène se produit lorsque l'écrivain, le narrateur ou le personnage est complètement bilingue et peut déterminer quels mots et quelles structures des deux langues expriment le mieux l'idée qu'il veut transmettre.

Les premiers paragraphes de "Pollito/Chicken》 illustrent comment l'emprunt, le transfert et le code-switching peuvent être manipulés en même temps : "I really had a wonderful time, dijo Suzie Bermiudez a su jefe tan pronto puso un spike heel en la oficina. He responds San Juán is wonderful, corroboró el jefe [...], reprimiendo ferozmente el deseo de añadir : I wonder why you Spicks don't stay home and enjoy it » (Vega, 1981, p. 75). Dans un seul passage le langage original comprend l'emprunt - «spike heel », le code switching : «I really had a wonderful time, dijo[...] tan pronto como 
puso un spike heel en la oficina "; et le transfert: le jeu de mots des mots apparentés « spike » et « Spick».

Nous nous sommes efforcées de traduire les nouvelles de Vega pour pouvoir communiquer à un public restreint l'opacité et la complexité de la multi-langue et de la multi-culture de Porto Rico. Mais la traduction sert plutôt de brouillon pour une discussion qui en principe rend les nouvelles dans leur forme originale plus compréhensibles.

Pour les nouvelles en espagnol et en français, la traduction vise à garder, à imiter la richesse linguistique de la nouvelle, à trouver un langage qui démontre une richesse parallèle au mélange de cultures antillaises : voilà la diversité de l'Éloge de la créolité qui est aussi à l'ordre du jour pour la traduction des Antilles.

À l'égard du créole incorporé dans le français antillais, il serait idéal de poursuivre le développement d'une autre interlangue anglaisfrançais-créole à la syntaxe africaine (et dont le vocabulaire comprendrait quelques éléments de l'espagnol aussi?) qui s'approcherait respectueusement du gouffre de la vie originale du texte ou de l'avant-le-texte oral et vécu.

Le traducteur a le droit, et peut-être la responsabilité, de communiquer cette richesse et cette subtilité aux lecteurs anglophones, surtout là où l'isolement ethnocentrique et colonisateur d'une " monoculture " - que les lecteurs sont d'ailleurs prêts à abandonner avec l'articulation d'une nouvelle direction - exige que nous fêtions les Antilles en les traduisant selon le vœu des auteurs antillais qui ont choisi de publier pour le plus grand public en écrivant en français martiniquais et en espagnol portoricain.

Université Humboldt State

\section{Références}

ANTOINE, Régis (1992). La littérature franco-antillaise: Haïti, Guadeloupe et Martinique. Paris , Karthala, $2^{\mathrm{e}}$ édition 1995.

BENJAMIN, Walter (1997). « L'abandon du traducteur. Prolégomènes à la traduction des "Tableaux parisiens " de Charles Baudelaire ». Trad. Laurent Lamy et Alexis Nouss. TTR, volume X, n², pp. 13-69.

BERNABÉ, Jean, CHAMOISEAU, Patrick et Raphaël CONFIANT (1989). Éloge de la créolité. Paris, Gallimard. 
BUDICK, Sanford (1996). «Crises of Alterity: Cultural Untranslatability and the Experience of Secondary Otherness ». The Translatability of Cultures. Robert Folkenflik, ed. Stanford, Stanford UP, pp. 1-22.

CHAMOISEAU, Patrick (1992). Texaco. Paris, Gallimard.

DRACIUS, Suzanne (1992). "De sueur, de sucre et de sang ". Le Serpent à Plumes, 15, pp. 35-38.

- (1995). «La Virago ». DIVERSITÉ : La nouvelle francophone à travers le monde. Boston, Houghton Mifflin, pp. 74-78.

- (1996). «The Virago ». Trad. Doris Y. Kadish et Jean-Pierre Piriou. Callaloo, vol. $19, \mathrm{n}^{\circ} 1, \mathrm{pp}$. 143-147.

- (2000). «La Montagne de Feu ». DIVERSITÉ: La nouvelle francophone à travers le monde. Boston, Houghton Mifflin, $2^{\mathrm{e}}$ édition. pp. 68-75.

FANON, Frantz (1952). Peau noire, masques blancs. Paris, Seuil.

FONKOUA, Romuald (1992). "Panorama de la nouvelle antillaise". Notre Librairie: La Nouvelle: Afrique noire, Maghreb, Caribes, Océan Indien. $\mathrm{N}^{\circ} 111$, oct.-déc., pp. 68-82.

GLISSANT, Édouard (1981). Le discours antillais. Paris, Seuil.

- (1990). Poétique de la relation. Paris, Gallimard.

GUINESS, Gerald (1984). "Images in Contemporary Puerto Rican Literature ». Ed. Adela Rodríguez de Laguna. The Puerto Ricans in Two World Contexts. New Brunswick, Transaction.

LEFEVERE, André (1977). Translating Literature: The German Tradition from Luther to Rosezweig. Assen, Van Gorcum.

LEVINE, Suzanne Jill (1991). Subversive Scribe: Translating Latin American Fiction. St. Paul, Graywolf Press.

Notre Librairie (1992). La Nouvelle: Afrique noire, Maghreb, Caraïbes, Océan Indien. $\mathrm{N}^{\circ} 111$, octobre-décembre, pp. 68-82.

POPLACK, Shana (1982). "'Sometimes I'll start a sentence in Spanish y termino en español' : toward a typology of code-switching ". Eds. Jon Amastae et Lucía Elías-Olivares. Spanish in the United States: Sociological Aspects. NY, Cambridge UP, pp. 230-263. 
ROBINSON, Douglas (1991). The Translator's Turn. Baltimore, Londres, Johns Hopkins University Press.

VEGA, Ana Lydia et FILIPPI, Carmen Lugo (1981). Vírgenes $y$ mártires. Rîo Piedras, PR, Antillana.

- «Historia de arroz y habichuelas ». Transmise par l'auteure à l'éditrice Marjorie Agosín pour inclusion dans Alchemy of the Palate (inédite). Université de Wellesley, Massachusetts.

- "A story of Rice and Beans ». Trans. Janet Sprina-Cunningham. (Inédite), sous contrat avec White Pine Press.

VELEZ, Diana (1986). «Pollito/Chicken: Split Subjectivity, National Identity \& the Articulation of Female Sexuality in a Narrative by Ana Lydia Vega ". The Americas Review, vol. 14, n² 2, pp. 68-76.

RÉSUMÉ : Traduire les Antilles en anglais: Trahir ou fêter la polyphonie? - Les rapports complexes de langue-culture aux Antilles, lorsqu'on les traduit du français vers l'anglais, se situent sur les plans culturel, naturel et créole-francais. Quand on traduit l'espagnol de Porto Rico en anglais, il s'agit surtout de problèmes d'emprunts, de transfert et de code switching anglais-espagnol dans l'original. Dans les deux cas, par contre, la même question se pose pour le traducteur: comment fêter la polyphonie sans la trahir? Nous examinons quatre nouvelles martiniquaises et portoricaines et leur unicité de contexte pour offrir des solutions spécifiques, des expressions exemplaires, des textes et contextes problématiques, et aussi pour signaler certains problèmes difficiles que pose la traduction des Antilles.

ABSTRACT : Translating the Caribbean into English: to Betray or to Celebrate Polyphony? - Translating the complex languageculture relationships in Caribbean French to English involves issues of culture, nature, and the Creole influence on the French language. In translating Puerto Rican Spanish to English, however, the linguistic issues are more often those of borrowing, transfer and code switching English-Spanish. In both cases the same question arises : how can the translator celebrate the polyphony of the Caribbean without betraying it? We look at four short stories from Martinique and Puerto Rico and, considering their unique contexts, offer specific solutions, exemplary expressions, problematic texts and contexts, and more difficult problems posed by the translation of the Caribbean.

Key words: short story, Puerto Rico, Martinique, Ana Lydia Vega, Suzanne Dracius.

Mots-clés : nouvelle, Porto Rico, Martinique, Ana Lydia Vega, Suzanne Dracius. 
Valérie Budig-Markin : Département de langues modernes, Programme de français, Humboldt State University, Arcata, CA 95521.

Courriel : budigmar@humboldt.edu

Martha Manier : Département de langues modernes, Programme d'espagnol, Humboldt State University, Arcata, CA 95521.

Courriel : mjm4@humboldt.edu 